\title{
Dual-Band Circularly Polarized Microstrip Antenna for GPS Application
}

\author{
Takafumi FUJIMOTO†*, Daisuke AYUKAWA $\ddagger$, \\ Kouhei IWANAGA $\dagger$, Mitsuo TAGUCHI $\ddagger$ \\ $\dagger$ Graduate School of Science \& Technology, Nagasaki University \\ † Dept. of Electrical \& Electronic Eng., Nagasaki University \\ 1-14 Bunkyo-machi, Nagasaki-shi, 852-8521, Japan \\ E-mail: takafumi@nagasaki-u.ac.jp
}

\section{Introduction}

Global positioning system (GPS) is one of the intelligent transport system (ITS) applications. The GPS antenna operates at both the $L 1$ and $L 2$ bands $(1.575 \mathrm{GHz}$ and $1.227 \mathrm{GHz}$ ). In a mobile communication system such as the GPS, the car antenna is required to be as small as possible.

In this paper, the small dual-band circularly polarized square MSAs for GPS application is proposed and the operational principles and the characteristics of the antenna are clarified by the simulator. The antenna consists of a square patch with slits and four $T$-shaped elements. The $T$-shaped element is loaded at each slit of the square patch. Yang et al. have proposed small dual-band circularly polarized square MSA with four $T$-shaped elements at edges of the square patch and shown the measured antenna characteristics [1]. The antenna proposed in [1] operates at about two resonant frequencies of TM10 and TM30 modes. Therefore, it is difficult that the ratio of the two frequencies giving the best axial ratio decreases to apply the antenna to the GPS (the ratio of two frequencies of GPS=1.284). The frequency ratio of the antenna in [1] is approximately 1.7 .

In the calculations in this paper, the simulator software package IE3D 10.2 based on the method of moments in the spectral domain [2] is used.

\section{Geometry of Antenna}

Fig. 1 shows a dual-band circularly polarized square MSA for GPS application. The width of the square patch is $W_{T}$. In order to achieve right handed circular polarization in the dual bands, the widths of the $T$-shaped elements along the $x$ axis are wider than those along $y$ axis, $d_{b}>d_{c}$, and the lengths are both $d_{a}$. The width of the slits between the square patch and $T$-shaped elements is $S_{t}$.

The relative dielectric constant and thickness of the dielectric substrate are $\varepsilon_{r}=2.60$ and $h=2.4 \mathrm{~mm}$, respectively. The antenna is excited at $x_{0}, y_{0}$ around the diagonal on the patch by a coaxial feeder through the dielectric substrate. 


\section{Results and Discussion}

\subsection{Parameter Study}

Fig. 2-4 show the lower frequency $f_{L}$, higher frequency $f_{H}$ and the ratio of $f_{H}$ to $f_{L}$ for changes of the parts of the $T$ element, $d_{a}, d_{W}$ and $d_{L}$, respectively. In each parameter, the ratio of the dimension of the $T$-shaped elements $d_{b} / d_{c}$ is adjusted to obtain the best axial ratio at two frequencies. $f_{L}$ and $f_{H}$ decrease as the length $d_{a}$ increases, moreover, $f_{H} / f_{L}$ also decreases. Similarly, as the length $d_{L}$ increases, $f_{L}$ and $f_{H}$ decreases. Although, however, the width $d_{W}$ influences $f_{L}$, it does not affect $f_{H}$. These characteristics denote that the dimensions of $T$-shaped elements influence $f_{L}$ and the lengths of the $L$-shaped slits between the $T$-shaped elements and the square patch influence $f_{H}$.

Fig. 5 shows the intensity of the time averaged electric current distribution at $f_{L}$ and $f_{H}$. At both $f_{L}$ and $f_{H}$, the electric currents flow around the $L$-shaped slits and $T$-shaped elements strongly. The $T$-shaped elements operate as main radiation elements at the lower frequency and the $L$-shaped slits operate as main radiation elements at the higher frequency.

As mentioned above, the width of the $T$-shaped elements $d_{W}$ don't influence the the frequency $f_{L}$. The fact makes the designing of the antenna easier. In the design procedure, at first the size of the patch $W_{T}, d_{L}, d_{a}$ and $d_{b} / d_{c}$ of the $T$-shaped elements are adjusted to tune the antenna to the high band and after that the width of the $T$-shaped elements $d_{W}$ is adjusted to tune the antenna to the low band.

\subsection{Design of GPS Antenna}

In the GPS, two bands, $L 1$ and $L 2$ bands, are used. The center frequencies of the $L 1$ and $L 2$ bands are $1.575 \mathrm{GHz}$ and $1.227 \mathrm{GHz}$, respectively. The bandwidth is $24 \mathrm{MHz}$ and the right handed circular polarization is used in both bands. The antenna for GPS application is designed using the design procedure stated in the previous section. Fig. 6(a) and (b) show the return loss and axial ratio, respectively. The bandwidths (return loss $\leqq-10 \mathrm{~dB}$ with axial ratio $\leqq 3 \mathrm{~dB}$ ) are very narrow (approximately $2 \mathrm{MHz}$ ) in both bands. This is due to the fact that the thickness of the dielectric substrate is much smaller than the wavelength in the dielectric.

\section{Conclusion}

A square MSA with four T-shaped elements for dual band operation in GPS application has been proposed. The operational principles of the antenna and the design procedure of the antenna for GPS application was discussed. Moreover, the dual-band antenna for GPS was designed using the design procedure.

\section{References}

[1] K. P. Yang, et al. , "Dual-band circularly-polarized square microstrip antenna, " IEEE Trans. Antennas and Propag., vol. 49, no. 3, pp.377-382, Mar. 2001.

[2] http://www.Zeland.com. 


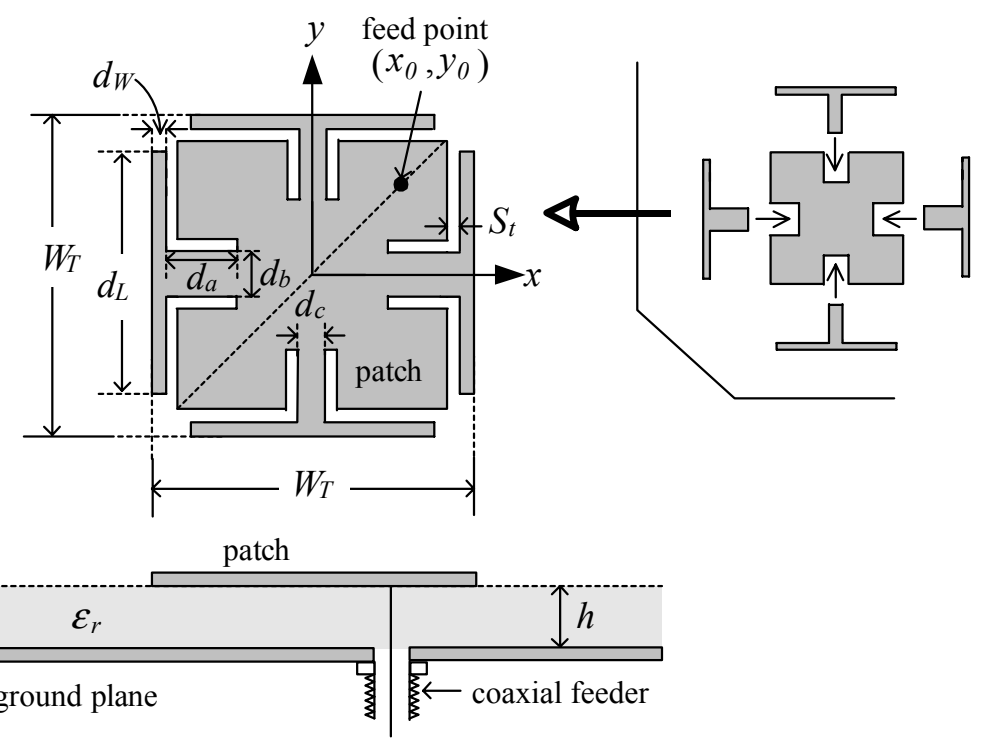

Fig. 1 Geometry of a square MSA with $T$ shaped elements

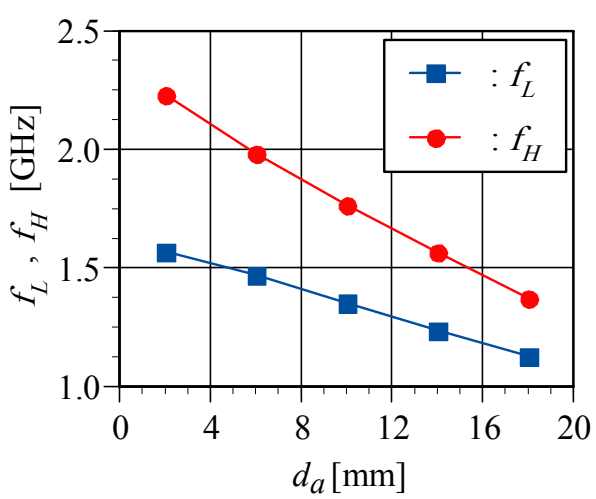

(a) $f_{L}, f_{H}$

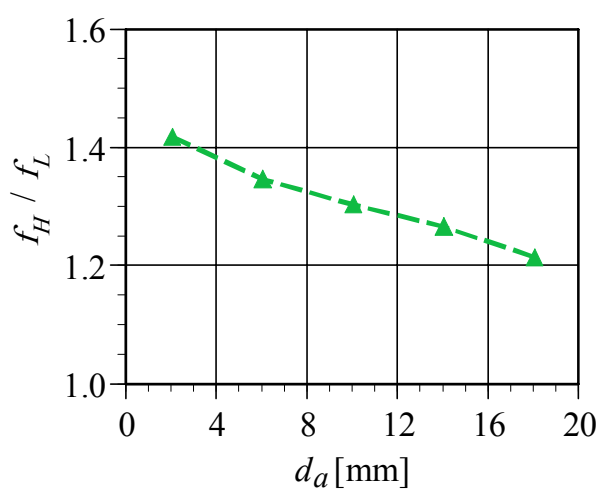

(b) $f_{H} / f_{L}$

Fig. 2 Two frequencies $f_{L}$ and $f_{H}$ and the frequency ratio $f_{H} / f_{L}$ against $d_{a}$ $\left(W_{T}=54 \mathrm{~mm}, d_{L}=48 \mathrm{~mm}, d_{W}=1.0 \mathrm{~mm}, S_{t}=0.5 \mathrm{~mm}\right)$

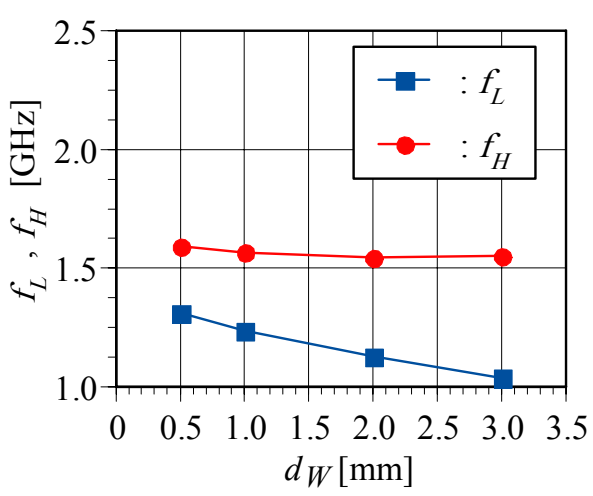

(a) $f_{L}, f_{H}$

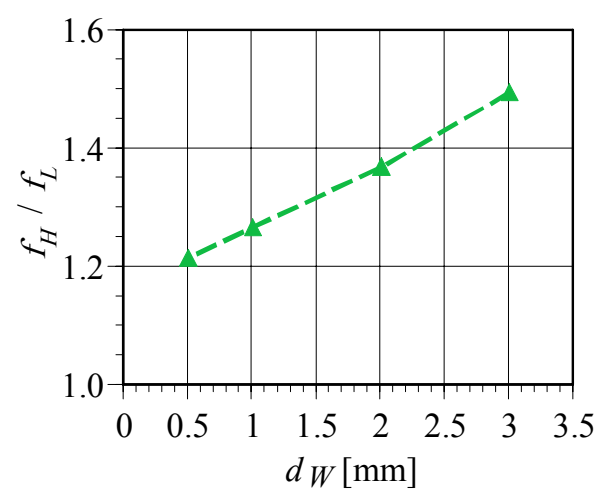

(b) $f_{H} / f_{L}$

Fig. 3 Two frequencies $f_{L}$ and $f_{H}$ and the frequency ratio $f_{H} / f_{L}$ against $d_{W}$ $\left(W_{T}=54 \mathrm{~mm}, d_{L}=48 \mathrm{~mm}, d_{a}=14.0 \mathrm{~mm}, S_{t}=0.5 \mathrm{~mm}\right)$ 


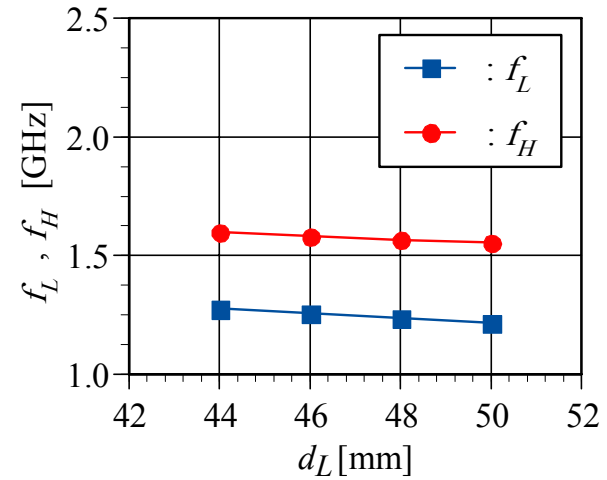

(a) $f_{L}, f_{H}$

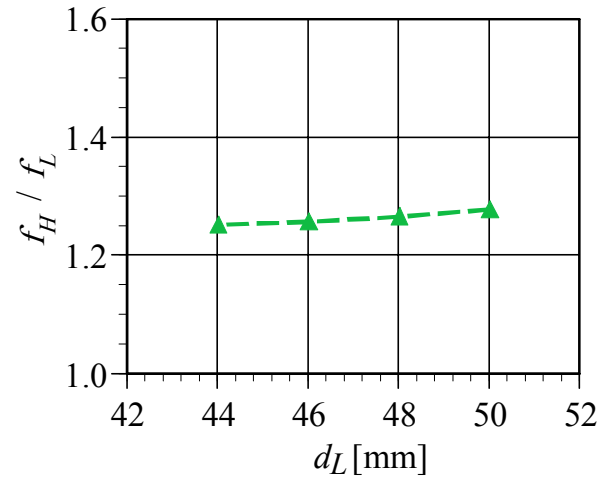

(b) $f_{H} / f_{L}$

Fig. 4 Two frequencies $f_{L}$ and $f_{H}$ and the frequency ratio $f_{H} / f_{L}$ against $d_{L}$ $\left(W_{T}=54 \mathrm{~mm}, d_{a}=14 \mathrm{~mm}, d_{W}=1.0 \mathrm{~mm}, S_{t}=0.5 \mathrm{~mm}\right)$

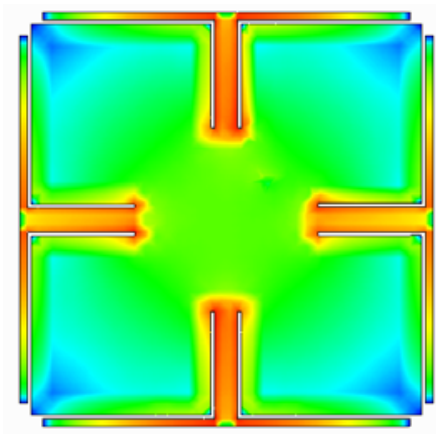

(a) $f_{L}=1.236 \mathrm{GHz}$

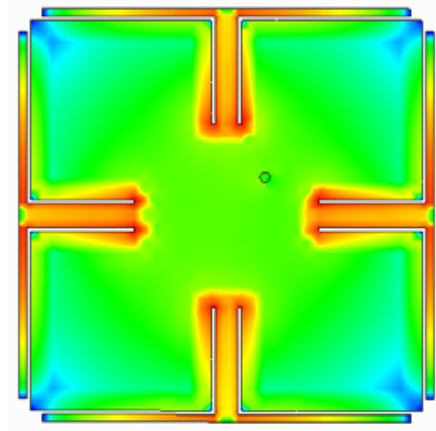

(b) $f_{H}=1.567 \mathrm{GHz}$

Fig. 5 Electric current distributions $\left(W_{T}=54 \mathrm{~mm}, d_{a}=14 \mathrm{~mm}, d_{W}=1.0 \mathrm{~mm}, d_{L}=48 \mathrm{~mm}, S_{t}=0.5 \mathrm{~mm}\right)$

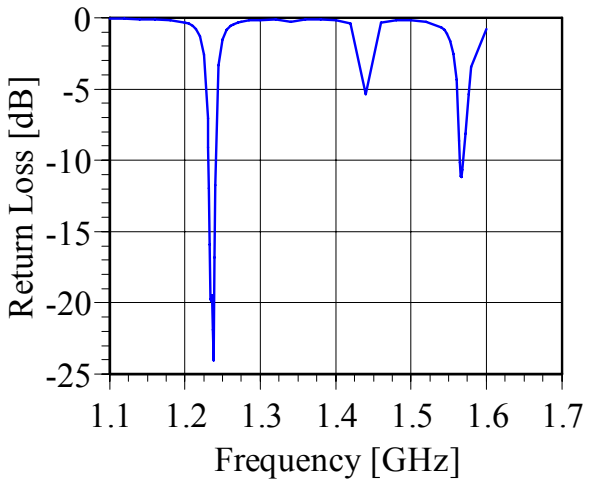

(a) return loss

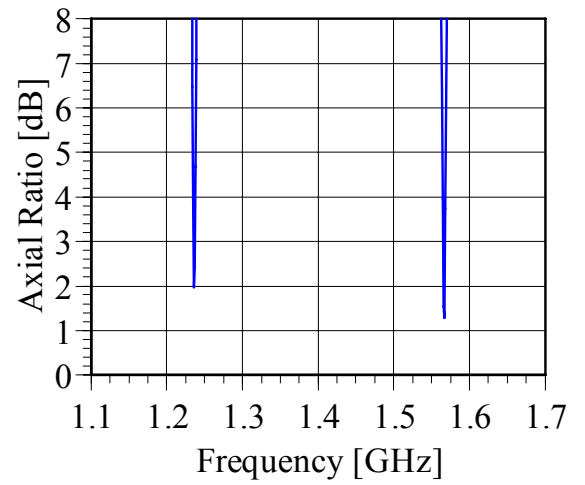

(b) axial ratio

Fig. 6 Return loss and axial ratio of GPS antenna $\left(W_{T}=54 \mathrm{~mm}, d_{a}=14 \mathrm{~mm}, d_{W}=1.0 \mathrm{~mm}, d_{L}=48 \mathrm{~mm}, S_{t}=0.5 \mathrm{~mm}\right)$ 